\title{
Health-related quality of life in surgical children and adolescents with congenital heart disease compared with their age-matched healthy sibling: a cross- sectional study from a lower middle-income country, Pakistan
}

\author{
Laila Akbar Ladak, ${ }^{1}$ Babar S Hasan, ${ }^{2}$ Janice Gullick, ${ }^{3}$ Khadija Awais, ${ }^{4}$ \\ Ahmed Abdullah, ${ }^{4}$ Robyn Gallagher ${ }^{1}$
}

- Additional material is published online only. To view, please visit the journal online (http://dx.doi.org/10.1136/ archdischild-2018-315594)

${ }^{1}$ Charles Perkins Centre and Susan Wakil School of Nursing and Midwifery, Sydney Nursing School, University of Sydney, Camperdown, New South Wales, Australia

${ }^{2}$ Department of Pediatrics and Child Health, The Aga Khan University, Karachi, Pakistan ${ }^{3}$ Susan Wakil School of Nursing and Midwifery, Sydney Nursing School, University of Sydney-Mallett Street Campus, Camperdown, New South Wales, Australia

${ }^{4}$ Medical College, The Aga Khan University, Karachi, Pakistan

\section{Correspondence to} Laila Akbar Ladak, Charles Perkins Centre, Susan Wakil School of Nursing and Midwifery, Sydney Nursing School, The University of Sydney, Camperdown NSW 2006 Australia;

llad8915@uni.sydney.edu.au

Received 24 May 2018 Revised 4 October 2018 Accepted 4 October 2018 Published Online First 15 December 2018
ABSTRACT
Objective Inconsistencies have been reported in health-related quality of life (HRQOL) in postoperative congenital heart disease (CHD). Despite the need for lifelong care due for residual symptoms, only a few studies have explored cardiac-related HRQOL but none in lower middle-income countries (LMIC). This study therefore addresses the gap by exploring HRQOL and its associated predictors in postoperative CHD in Pakistan. Outcome measures General and cardiac-related HRQOL, associated predictors.

Methods This cross-sectional study recruited patients with CHD and age-matched healthy siblings as controls ( $n=129$ each) at a single centre in Pakistan. Patients and their siblings completed HQROL surveys (PedsQL 4.0 Generic Core, PedsQL Cognitive Functioning). Patients only completed PedsQL 3.0 Cardiac module. Generalised linear models identified predictors.

Results The sample mean age was $8.84 \pm 3.87$ years and $70 \%$ were below the poverty line for an LMIC. The majority $(68 \%)$ had their first surgery after 1 year of age and were interviewed at a mean $4.08 \pm 1.91$ years postoperatively. Patients with CHD had lower HRQOL in all domains compared with their age-matched siblings, with the biggest differences for total HRQOL (effect size, $d=-1.35)$. Patients with complex CHD had lower HRQOL compared with simple to moderate CHDs in cardiacrelated HRQOL. The lowest scores were for treatment problems (effect size, $d=-0.91$ ). HRQOL was worse for patients who were on cardiac medications, had complex $\mathrm{CHD}$, longer cardiopulmonary bypass time, re-operations and were female.

Conclusions HRQOL issues persist in postoperative patients with CHD in LMIC, Pakistan. Solutions are needed to address poor HRQOL and lifelong concerns of patients and their parents.

\section{Check for updates}

(C) Author(s) (or their employer(s)) 2019. No commercial re-use. See rights and permissions. Published by BMJ.

To cite: Ladak LA, Hasan BS, Gullick J, et al. Arch Dis Child 2019:104:419-425.

\section{INTRODUCTION}

While advances in congenital heart disease (CHD) surgical techniques and operative care have decreased postoperative (postop) mortality and improved health-related quality of life (HRQOL), often residual issues can have long-term negative effects. ${ }^{1}$ HRQOL is an important outcome for patients and families and both a predictor of

\section{What is already known on this topic?}

- Benefits of surgery for patients with congenital heart disease (CHD) are not fully realised in health-related quality of life (HRQOL) compared with unaffected children and adolescents.

- It is unclear whether HRQOL outcomes are similar in lower middle-income countries (LMIC), considering the differences in the available resources.

\section{What this study adds?}

- This is the first study from an LMIC to explore both general and cardiac-related HRQOL and its associated predictors in surgical children and adolescents with CHD.

- Both general and cardiac HRQOL are poor in surgical patients with CHD compared with agematched healthy siblings in Pakistan and worse outcomes are associated with clinical and sociodemographic factors.

- HRQOL assessment should be part of clinical assessment of CHD surgical outcomes particularly in LMIC.

mortality and hospitalisation as well as an indicator of future resource allocation requirements. ${ }^{2}$

HRQOL in CHD compared with healthy controls has been reported; however, there have been inconsistencies in the findings. For instance, impaired HRQOL has been reported in physical, ${ }^{34}$ emotional, ${ }^{5}$ school,${ }^{56}$ cognitive, ${ }^{7}$ psycholog$\mathrm{ical}^{8}$ domains and overall HRQOL, ${ }^{5}$ whereas other reports indicate equal or even better HRQOL in these domains. ${ }^{6-11}$ Importantly, the cardiac state of patients with CHD influences HRQOL, ${ }^{478}$ yet few papers focus on cardiac-specific HRQOL. A recent meta-analysis reported lower cardiac-related HRQOL in CHD, particularly in complex CHD compared with moderate and simple severity. ${ }^{12}$

The role of resources in HRQOL outcomes for patients with CHD should not be ignored, and as the majority of research has been conducted 
in high-income countries (HIC), this is an important deficit. ${ }^{12}$ Given the differences in culture, education, sociodemographics and the availability of healthcare services, the results for HIC may not apply to lower middle-income countries (LMIC). Despite the huge burden of CHD in LMIC, there is dearth of data from this region. ${ }^{12}$ This study was conducted in Pakistan and aimed to compare HRQOL in post-op patients with CHD to their age-matched healthy sibling and identify the associated predictors (preoperative, intraoperative, postop and postdischarge, sociodemographics) of HRQOL in these patients.

\section{METHODS}

The study protocol has been published ${ }^{13}$ and an overview is provided below. The study adhered to the ethical guidelines of the 1975 Declaration of Helsinki.

\section{Study design}

The study used a cross-sectional study design to compare HRQOL between patients with CHD and their age-matched siblings, and between CHD severities for cardiac-specific HRQOL in patients with CHD. The Wilson and Cleary model (see online supplementary figure 1) provided a guiding framework for this study. ${ }^{14}$

\section{Study setting}

The study was conducted at The Aga Khan University Hospital, Pakistan, which is a 1400 bed tertiary care hospital. The facility has patient representation from all the states of Pakistan including both urban and rural populations. Paediatric cardiac surgery was first established in 2008 using a multidisciplinary team including surgeon, intensivist, cardiologist, nurse and physiotherapist. Approximately 40 CHD surgeries (simple, moderate and complex severities) are performed per month. CHD has been categorised for the study based on severity as simple, moderate and complex severity. ${ }^{15}$ For example, repaired secundum or sinus venosus atrial septal defect without residua is classified as simple, coarctation of the aorta and tetralogy of Fallot as moderate and Fontan procedure and transposition of the great arteries as complex CHD severity ${ }^{1516}$ (see online supplementary appendix A).

\section{Subjects}

Patients were eligible for the study if they had CHD surgery at the study site from the introduction of surgery in June 2008 to July 2015, were at least 1 year postsurgery and were able to understand English and/or Urdu. Patients were excluded if, in addition to CHD, they had any chromosomal abnormality due to the potential confounding influence on HRQOL. ${ }^{10}$ An age-matched healthy sibling was used as control for each patient with CHD enrolled to adjust for potential genetic and sociodemographic confounders, consistent with previous work in the area. ${ }^{17}$ As family size in Pakistan is typically four to five children, who often accompany patients to clinic visits, recruiting age-matched siblings was very effective. Siblings within the closest age possible and a maximum range of 2-3 years of the patient's age were recruited as controls. ${ }^{18}$

Sample size was calculated to be a minimum of 95 per group based on a small Cohen's d effect size (0.2) in HRQOL between the patient with CHD and their age-matched sibling, a power of 0.8 , a probability of 0.05 and 11 predictors. ${ }^{19}$

\section{Procedure}

A stratified random sample was identified based on the patients' representation from each surgical year since the study site initiated its paediatric cardiac surgery in 2008 till the study's data collection period, that is, 2015 ( $\sim 75$ patients/year, 600/1050 eligible patients). Patients, their age-matched healthy siblings and parents were sent study invitations and a follow-up phone call (see online supplementary figure 2). Of the 600 invitations, 310 were returned, 38 refused and 252 patients were recruited. This paper focuses on the HRQOL of patients aged 2-18 years $(n=129)$, their age-matched siblings $(n=129)$ and parents.

\section{Data collection \\ Health-related quality of life}

Informed consent was obtained from parents and assent from patients (aged 5-18 years). The PedsQL questionnaire was used to explore HRQOL in surgical patients with CHD. This questionnaire focusses on the domains identified for this study, have been widely used in research studies, ${ }^{6} 1018$ 20-23 have well-established validity and reliability ${ }^{24}$ and are age specific.

General HRQOL was assessed through PedsQL 4.0 Generic Core Scale, which has 23 items and focusses on domains of physical, emotional, social and school functioning (see online supplementary appendix B). The PedsQL Cognitive Functioning Scale was used to explore cognitive functioning and has six items. Disease-specific HRQOL was measured through the PedsQL 3.0 Cardiac Module, which is a 27-item scale and includes heart and treatment problems, perceived physical appearance, treatment anxiety, cognitive and communication problems. Patients were asked to provide scores for the HRQOL questions on their past one month's condition, from zero to four with zero 'never a problem' and four 'almost always a problem'. Responses were reverse scored and linearly transformed to 0-100 with higher scores indicating better HRQOL. Permission was sought from Mapi Research Trust to use the questionnaires, and translation into Pakistan's national language of Urdu, abiding to the translation protocol. ${ }^{25}$ Parental responses substituted for children under 5 years of age $(n=22)$ and 20 children did not attend school so school functioning data were not completed.

We adopted a minimal clinically meaningful difference between CHD and control of 4.4 score points on the total HRQOL. ${ }^{26}$ The reliability of the questionnaires was found to be good for all domains (Cronbach's $\alpha=0.825-0.937$ ).

\section{Clinical and sociodemographic variables}

Sociodemographic data were collected using a checklist and included age, gender, socioeconomic background (World Bank's poverty line definition of US $\$ \leq 2 /$ day $\mathrm{LMIC}),{ }^{27}$ educational status, occupation and family structure. Clinical data included current cardiac medications and New York Heart Association functional class. CHD diagnosis severity (simple, moderate, complex), ${ }^{15}$ age at diagnosis and surgery, type and number of surgical procedure, cardiopulmonary bypass time (CBT), Risk Adjustment for Congenital Heart Surgery (RACHS 1) score ${ }^{28}$ and length of stay (LOS) were retrieved from the patient's medical record.

\section{Statistical analysis}

Data were analysed using the Statistical Package for Social Sciences (IBM SPSS Statistics 22). Means and SD and/or median and IQR were calculated for continuous variables and frequencies were calculated for categorical variables. There were no missing data.

Differences in HRQOL between patients with CHD and their age-matched siblings were calculated using two-sided paired $\mathrm{t}$-tests with a $\mathrm{p}$ value of $<0.05$ as statistically significant. Effect 
size was calculated using Cohen's d to identify the magnitude of HRQOL difference on PedsQL questionnaires. A Cohen's d of 0.2 was considered small, 0.5 as medium and 0.8 as a large effect size. $^{29}$

Generalised linear models (GLM) were used to explore the relationship between general and cardiac-specific HRQOL scores and clinical/sociodemographic variables. Differences in mean scores between patients with CHD and siblings were calculated for each domain of HRQOL and were included as a dependent variable in the model. A separate model was created for cardiac-specific HRQOL domains. A total of 11 independent variables identified in the literature were selected to include in models for both general as well as cardiac-related HRQOL and included age at surgery, CBT, LOS, number of operations, RACHS scale, current cardiac medications, gender, parental education, socioeconomic background and family structure. Linearity, homoscedasticity (validating homogeneity of variance using scatter plots of residuals and predictors, with the assumption that all the data points are close to the regression line), multicollinearity and normality of residuals were checked to ensure the data met the GLM required assumptions. ${ }^{30}$ If the graphs tended to form a funnel-shape at the higher end of the predicted values, it was an indicator of heteroscedasticity (unequal variance across the different levels of the independent variables). Therefore, weighted GLM analysis was performed based on the assumption that by adding the weights (least square of the variance), homoscedasticity variance of the predicting variables will be achieved across the regression line. ${ }^{31}$ A weighted GLM analysis was conducted to address the heteroscedasticity for total HRQOL, all GLM models were found to be plausible for all the domains.

\section{RESULTS}

Patients with CHD had a mean age of $8.84 \pm 3.87$ years and were more often male $(n=87,67 \%)$ (table 1$)$. The majority $(n=106$, $82 \%$ ) of participants were from Karachi, which is Pakistan's most populous city. Most patients $(n=74,57 \%)$ lived in extended families and half of the patients' parents had high school education. The majority of families $(n=90,70 \%)$ were living below the poverty line for LMIC.

Most patients with CHD $(n=85,66 \%)$ had moderate CHD severity and about a third $(n=36,28 \%)$ had complex $\mathrm{CHD}^{15}$ (table 1$)$. The majority of the patients $(n=107,83 \%)$ were diagnosed by 1 year of age and mean follow-up postsurgery was $4.08 \pm 1.91$ years.

General HRQOL scores were significantly lower in the CHD cohort compared with their healthy siblings across all domains (table 2). There was a clinically meaningful difference of 12.81 points $^{26}$ in total HRQOL between the groups, with significantly lower HRQOL for patients with CHD in total (effect size, $d=-1.35$ ), emotional (effect size, $d=-1.29$ ), psychosocial (effect size $d=-1.28$ ), physical (effect size, $d=-1.10$ ), school functioning (effect size $\mathrm{d}=-0.96$ ), social (effect size, $\mathrm{d}=-0.48$ ) and cognitive (effect size, $\mathrm{d}=-0.47$ ) functioning. HRQOL was significantly lower in complex versus simple-to-moderate $\mathrm{CHD}$ in cardiac-specific HRQOL in cardiac-related treatment (effect size, $\mathrm{d}=-0.91$ ), heart problems (effect size, $\mathrm{d}=-0.66$ ) and communication problems (effect size, $\mathrm{d}=-0.46$ ) (table 3 ).

The significant independent predictors for HRQOL in surgical patients with CHD are detailed in table 4 for general and table 5 for cardiac-related HRQOL. Prescription of cardiac medications was a strong predictor of worse HRQOL across almost all domains including physical (B -12.67, 95\% CI -4.01 to-21.32),
Table 1 Sociodemographic and clinical characteristics of surgical pateints with $\mathrm{CHD}$

\begin{tabular}{|c|c|c|}
\hline & n (129) & $\%$ \\
\hline \multicolumn{3}{|l|}{ Gender } \\
\hline Male & 87 & 67 \\
\hline \multicolumn{3}{|l|}{ Age groups (years) } \\
\hline$<4$ & 12 & 9 \\
\hline $5-9$ & 67 & 52 \\
\hline $10-15$ & 43 & 33 \\
\hline $16-18$ & 7 & 6 \\
\hline \multicolumn{3}{|l|}{ Education } \\
\hline \multicolumn{3}{|l|}{ Currently studying } \\
\hline Preschool & 28 & 22 \\
\hline Primary (grade $1-5$ ) & 52 & 40 \\
\hline High school (grade 6-12) & 26 & 20 \\
\hline Currently not studying & 20 & 16 \\
\hline Completed: high school (grade 12) & 3 & 2 \\
\hline Residential place: Karachi & 106 & 82 \\
\hline Income: below poverty line (LMIC) & 90 & 70 \\
\hline \multicolumn{3}{|l|}{ Family structure } \\
\hline Nuclear & 55 & 43 \\
\hline Extended & 74 & 57 \\
\hline \multicolumn{3}{|l|}{ Parental education } \\
\hline At least one parent completed high education & 64 & 50 \\
\hline At least one parent completed University & 65 & 50 \\
\hline \multicolumn{3}{|l|}{ CHD severity } \\
\hline Simple & 8 & 6 \\
\hline Moderate & 85 & 66 \\
\hline Complex & 36 & 28 \\
\hline \multicolumn{3}{|l|}{ CHD categorisation } \\
\hline Biventricle & 123 & 95.34 \\
\hline Single ventricle & 6 & 4.65 \\
\hline \multicolumn{3}{|l|}{ Number of surgeries } \\
\hline 1 & 109 & 85 \\
\hline$\geq 2$ & 20 & 15 \\
\hline \multicolumn{3}{|l|}{ Cardiac prognostic severity } \\
\hline RACHS 1 & 8 & 7 \\
\hline RACHS 2 & 92 & 71 \\
\hline RACHS $\geq 3$ & 29 & 22 \\
\hline \multicolumn{3}{|l|}{ Age at diagnosis (years) } \\
\hline$\leq 1$ & 107 & 83 \\
\hline$>1$ & 22 & 17 \\
\hline
\end{tabular}

Age at first surgery (years)

\begin{tabular}{lcc}
$\leq 1$ & 41 & 32 \\
$>1$ & 88 & 68 \\
NYHA I & 120 & 93 \\
Cardiac medications $^{*}$ & 27 & 21 \\
& Mean \pm SD & \\
Current age (years) & $8.84 \pm 3.87$ & \\
Time since last surgery (years) & $4.08 \pm 1.91$ & \\
Cardiopulmonary bypass time (min) & $110.14 \pm 50.71$ & \\
Length of stay postsurgery (days) & $6.74 \pm 3.26$ & \\
\hline
\end{tabular}

* Medications alone or in combination include ACE inhibitors, $\beta$-blocker, anticoagulant. CHD, congenital heart disease; NYHA, New York Heart Association; RACHS, risk adjustment for congenital heart surgery.

emotional ( $\mathrm{B}-10.88,95 \% \mathrm{CI}-1.87$ to -19.90$)$, psychosocial (B $-6.32,95 \%$ CI -0.48 to -12.17 ), total HRQOL (B -8.03 , 95\% CI -2.17 to -13.89 ), heart problem (B $-10.34,95 \% \mathrm{CI}$ -1.93 to -18.75$)$ and cognitive problem (B $-7.66,95 \% \mathrm{CI}$ -0.16 to -15.17$)$. However, patients prescribed cardiac medications had lesser communication problems (B 20.91, 95\% CI 38.52 to 3.30). Patients with complex CHD had lower HRQOL 
Table 2 General HRQOL in surgical patients with CHD vs age-matched siblings

\begin{tabular}{|c|c|c|c|c|c|}
\hline \multirow[b]{2}{*}{ Domains } & \multicolumn{2}{|c|}{ Patients with CHD $(n=129)$} & \multicolumn{2}{|c|}{ Siblings $(n=129)$} & \multirow[b]{2}{*}{ Cohen's d } \\
\hline & Mean \pm SD & $95 \% \mathrm{Cl}$ & Mean \pm SD & $95 \% \mathrm{Cl}$ & \\
\hline Physical* & $83.57 \pm 19.50$ & 80.57 to 86.97 & $98.95 \pm 3.28$ & 98.38 to 99.52 & -1.10 \\
\hline Emotional* & $73.91 \pm 17.32$ & 70.89 to 76.93 & $92.65 \pm 11.18$ & 90.69 to 94.57 & -1.29 \\
\hline Social* & $95.11 \pm 10.80$ & 93.20 to 96.95 & $99.14 \pm 4.65$ & 98.33 to 99.95 & -0.48 \\
\hline School* & $86.23 \pm 18.08$ & 83.27 to 90.12 & $98.81 \pm 3.32$ & 97.73 to 99.36 & -0.96 \\
\hline Psychosocial* & $84.88 \pm 12.11$ & 82.91 to 87.12 & $96.73 \pm 4.94$ & 95.85 to 97.58 & -1.28 \\
\hline Total HRQOL* & $84.49 \pm 12.72$ & 82.43 to 86.85 & $97.30 \pm 4.05$ & 96.58 to 98.00 & -1.35 \\
\hline Cognitive* & $90.49 \pm 18.41$ & 87.37 to 93.76 & $97.36 \pm 8.68$ & 95.87 to 98.89 & -0.47 \\
\hline
\end{tabular}

${ }^{*} \mathrm{P}<0.05$.

CHD, congenital heart disease; HRQOL, health-related quality of life.

in physical functioning ( $\mathrm{B}-9.03,95 \% \mathrm{CI}-17.59$ to -0.47 ) and perceived physical appearance (B -16.92, 95\% CI -31.67 to -2.18$)$. Longer CPBT was associated with lower HRQOL in perceived physical appearance (B $-0.12,95 \%$ CI -0.23 to -0.01$)$. Patients who had multiple surgical procedures had lower HRQOL for heart problems (B -10.60, 95\% CI -20.17 to -1.09$)$ and female patients had lower physical functioning $(-7.74,95 \% \mathrm{CI}-14.57$ to -0.91$)$.

\section{DISCUSSION}

This is the first study conducted in an LMIC comparing young postoperative patients with CHD with their age-matched siblings in general and cardiac-specific HRQOL. This study found that HRQOL was substantially lower in surgical patients with $\mathrm{CHD}$ in all domains compared with their age-matched healthy siblings, particularly for emotional, psychosocial, physical and school functioning. Disease severity was important. More complex CHD was associated with significantly lower cardiac-specific HRQOL, especially for heart and treatment-related problems. Also, surrogate markers like cardiac medications and longer CBT were important as more complex disease had worse HRQOL, even when many contextual and clinical variables were taken into account.

Poor HRQOL following CHD surgery in children and adolescents relative to healthy controls in LMIC is an important finding and is similar to the preponderance of reports from HIC. ${ }^{523} 32-34$ However, the findings are not consistent with a small group of studies. ${ }^{101133}$ These studies either focused on simple or moderate $\mathrm{CHD},{ }^{10}{ }^{11}$ had patients with early CHD surgery ${ }^{33}$ or had a longer follow-up period. ${ }^{1133}$ Early surgery is known to produce better outcomes as it prevents physiological compromise and cardiac remodelling. ${ }^{35}$ Moreover, patients may learn to cope and adapt to their disease over a longer follow-up time. ${ }^{36}$
The study findings highlight that the lowest HRQOL occurred in emotional functioning, which is a relatively universal issue across cultural and resource contexts. $5691820-2232$ This consistency suggests that the impact of CHD for children and adolescents has important emotional consequences, most likely related to the physiological and neurobiological changes that occur during this phase of life that influence emotional, cognitive and behavioural states. ${ }^{37}$ Adolescents are developing their self-concept and self-image when their symptoms may be visible and influential.

Female patients had worse outcomes, identifying an important group to target for intervention. Potential explanations for worse physical function in female include parent's tendency to overprotect children with chronic illness, which is then associated with poor HRQOL in children and adolescents. ${ }^{38}$ In a patriarchal society like Pakistan, parents are even more protective towards females which may lead to poor physical functioning. Furthermore, the CHD surgical scar may be more of an issue given the social expectations for a female in Pakistani society and has not been reported by studies conducted in HIC. ${ }^{39}$

Our study highlights the important role of clinical factors in HRQOL. All clinical predictors associated with lower HRQOL in our study were indicators of worse health and have been reported by others. 918202333 These factors have been proven to be counterbalanced by early detection, clinical management, preoperative, perioperative and postop care. ${ }^{40}$ However, in LMIC the resources available to address the needs of this population are scarce. In addition to the patient's personal profile, healthcare system and gross domestic product (country profile) have also been identified as strong predictors of HRQOL in patients with $\mathrm{CHD} .^{41}$

Considering the resource constraints in LMIC countries like Pakistan, HRQOL monitoring is critical as part of regular clinic

Table 3 Cardiac-related HRQOL in surgical patients with CHD

\begin{tabular}{|c|c|c|c|c|c|}
\hline \multirow[b]{2}{*}{ Domains } & \multicolumn{2}{|l|}{ Complex CHD } & \multicolumn{2}{|c|}{ Simple-to-moderate CHD } & \multirow[b]{2}{*}{ Cohen's d } \\
\hline & Mean \pm SD & $95 \% \mathrm{Cl}$ & Mean \pm SD & $95 \% \mathrm{Cl}$ & \\
\hline Heart problem* & $74.206 \pm 22.37$ & 66.63 to 81.77 & $87.211 \pm 15.97$ & 83.92 to 90.50 & -0.66 \\
\hline Treatment problem* & $89.56 \pm 14.46$ & 82.58 to 96.53 & $99.09 \pm 3.01$ & 97.06 to 101.11 & -0.91 \\
\hline $\begin{array}{l}\text { Perceived physical } \\
\text { appearance }\end{array}$ & $76.904 \pm 36.94$ & 64.21 to 89.59 & $86.51 \pm 26.56$ & 80.97 to 92.04 & -0.29 \\
\hline Treatment anxiety & $69.96 \pm 37.16$ & 57.39 to 82.54 & $80.30 \pm 30.72$ & 73.98 to 86.63 & -0.30 \\
\hline Cognitive problem & $91.33 \pm 15.30$ & 86.15 to 96.51 & $94.40 \pm 14.47$ & 91.42 to 97.38 & -0.20 \\
\hline Communication problem* & $53.8 \pm 38.63$ & 40.53 to 67.08 & $70.78 \pm 33.68$ & 63.85 to 77.72 & -0.46 \\
\hline
\end{tabular}

${ }^{*} \mathrm{P}<0.05$.

CHD, congenital heart disease; HRQOL, health-related quality of life. 


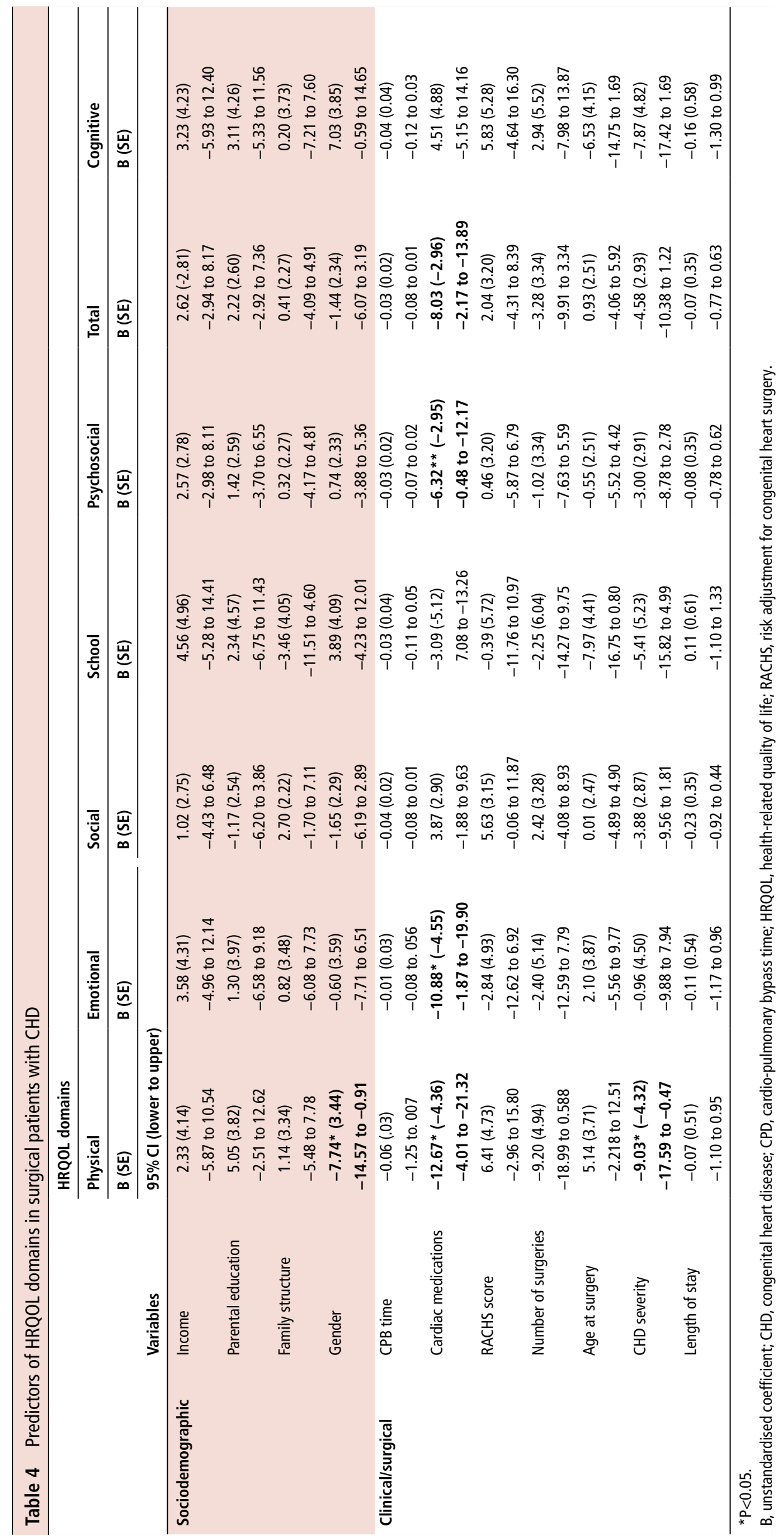


Table 5 Predictors of cardiac-related HRQOL in surgical patients with CHD

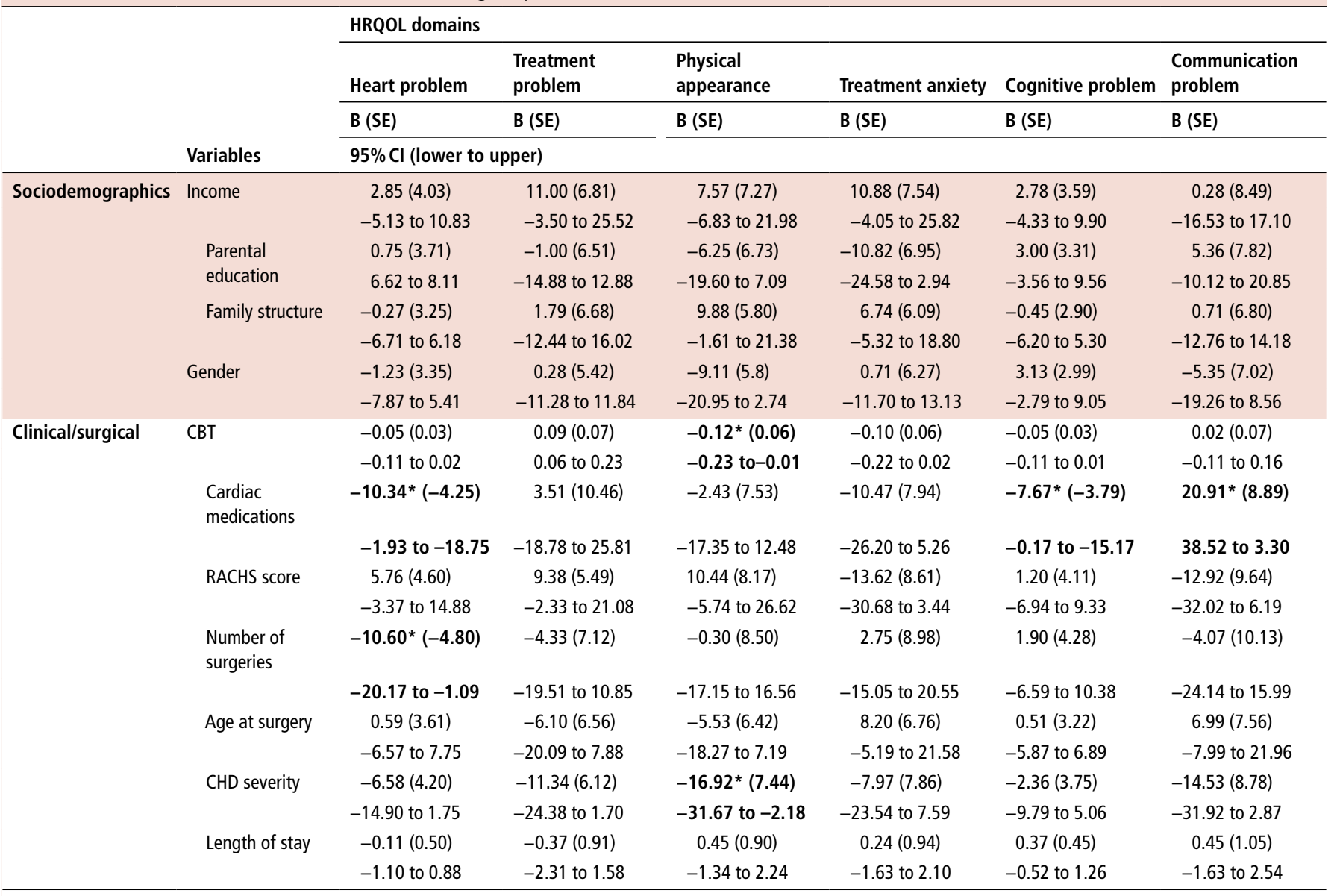

${ }^{*} \mathrm{P}<0.05$.

B, unstandardised coefficient; CHD, congenital heart disease; CPB, cardiopulmonary bypass time; HRQOL, health-related quality of life; RACHS, risk adjustment for congenital heart surgery.

assessments, allowing patients with $\mathrm{CHD}$ and parents, particularly those at high risk of poor HRQOL, to collaborate in the development of patient-centred care plans and early interventions. Since more children with CHD now survive to late adulthood, ${ }^{1}$ understanding parental concerns and issues will assist paediatric cardiologists and cardiac surgeons and associated multidisciplinary teams to support structured educational programmes and counselling for patients and parents to assist with realistic expectations, early nutrition, expected issues and early referrals following surgery. In addition, HRQOL provides a good benchmark for assessing the benefit of surgical and other therapeutic interventions, and reveals where the best patient-sensitive gains can be made.

The majority of our study participants were students and therefore school may be an important platform for intervention to improve HRQOL. The school health nurse model can be an efficient strategy in Pakistan as it has been shown to improve health condition management, promote mental health and leads to enhanced academic performance. ${ }^{42}$ Moreover, teachers can also be trained as health promoters who can address the health-specific needs of their students who have had CHD surgery in supporting their education and learning.

\section{Strengths and limitations}

A major strength of this study is that the study compared HRQOL in surgical patients with CHD with age-matched healthy siblings, controlling for several potential confounders that is, genetic, sociodemographic, familial and environmental. Second, this is one of the first HRQOL studies in LMIC setting and allows us to consider HRQOL in surgical patients with CHD in a resource-constrained environment.

Several limitations should be considered while interpreting the study findings. This was a single-centre study conducted in a private hospital; the sociodemographic profile of patients might differ from a public setting. Second, this cross-sectional study used a convenience sample of eligible patients willing to come for study interviews, there is a possibility of missing patients with particular CHD diagnoses. Third, this is the first time the PedsQL was used in this setting. Despite strictly abiding by the Mapi Research translation protocol (based on American Association of Orthopaedic Surgeons guidelines) for ensuring the accurate contextual translation, there could be a possible cross-culture bias.

Future research should focus on developing a HRQOL questionnaire, which is appropriate to the context of Pakistani society and culture. A multi-centre longitudinal study should be conducted recruiting patients from different parts of Pakistan and broader socio-demographic backgrounds to increase the generalisability of our findings.

\section{CONCLUSION}

HRQOL was poor in postop patients with CHD in Pakistan in comparison to their age-matched healthy siblings. Patients with complex CHD had worse cardiac-related HRQOL compared 
with simple-to-moderate CHD. HRQOL screening would be an important adjunct to routine care to highlight areas for intervention.

Contributors $\mathrm{LAL}$ is a PhD candidate who conceived the study, contributed to the study design, conducted the data collection, data analysis, drafted and revised the manuscript. BSH contributed to the study design, provided critical feedback on the manuscript drafts. JG contributed to the study design, provided critical feedback on the manuscript drafts. KA contributed to the study design, provided critical feedback on the manuscript drafts. AA contributed to the study design, provided critical feedback on the manuscript drafts. RG contributed to the study design, mentored in data analysis, provided critical feedback on the manuscript drafts. All authors approved the final manuscript as submitted and agreed to be accountable for all aspects of the work.

Funding This study is part of a PhD thesis. LAL received an International Postgraduate Research Scholarship (IPRS) and Australian Postgraduate Award (APA) from The University of Sydney, Australia to pursue Doctoral studies. This study has been endorsed by the International Society of Adult Congenital Heart Disease (ISACHD). However, no funding was secured for this study.

\section{Competing interests None declared.}

Patient consent Not required.

Ethics approval Ethics Review Committe, The Aga Khan University, Pakistan (3737-Ped-ERC-15).

Provenance and peer review Not commissioned; externally peer reviewed.

\section{REFERENCES}

1 Marelli AJ, lonescu-Ittu R, Mackie AS, et al. Lifetime prevalence of congenital heart disease in the general population from 2000 to 2010. Circulation 2014;130:749-56.

2 Gotay CC, Kawamoto CT, Bottomley A, et al. The prognostic significance of patientreported outcomes in cancer clinical trials. J Clin Oncol 2008;26:1355-63.

3 Fteropoulli T, Stygall J, Cullen S, et al. Quality of life of adult congenital heart disease patients: a systematic review of the literature. Cardiol Young 2013;23:473-85.

4 Kahr PC, Radke RM, Orwat S, et al. Analysis of associations between congenital heart defect complexity and health-related quality of life using a meta-analytic strategy. Int J Cardiol 2015;199:197-203.

5 Mellion K, Uzark K, Cassedy A, et al. Health-related quality of life outcomes in children and adolescents with congenital heart disease. J Pediatr 2014;164:781-8.

6 Tahirović $\mathrm{E}$, Begić $\mathrm{H}$, Tahirović $\mathrm{H}$, et al. Quality of life in children after cardiac surgery for congenital heart disease. Coll Antropol 2011;35:1285-90.

7 Karsdorp PA, Everaerd W, Kindt M, et al. Psychological and cognitive functioning in children and adolescents with congenital heart disease: a meta-analysis. J Pediatr Psychol 2007:32:527-41.

8 Latal B, Helfricht S, Fischer JE, et al. Psychological adjustment and quality of life in children and adolescents following open-heart surgery for congenital heart disease: a systematic review. BMC Pediatr 2009;9:6.

9 Landolt MA, Valsangiacomo Buechel ER, Latal B. Health-related quality of life in children and adolescents after open-heart surgery. J Pediatr 2008;152:349-55.

10 Kwon EN, Mussatto K, Simpson PM, et al. Children and adolescents with repaired tetralogy of fallot report quality of life similar to healthy peers. Congenit Heart Dis 2011;6:18-27

11 Mueller GC, Sarikouch S, Beerbaum P, et al. Health-related quality of life compared with cardiopulmonary exercise testing at the midterm follow-up visit after tetralogy of Fallot repair: a study of the German competence network for congenital heart defects. Pediatr Cardiol 2013;34:1081-7.

12 Ladak LA, Hasan BS, Gullick J, et al. Health-related quality of life in congenital heart disease surgery in children and young adults: a systematic review and meta-analysis. Arch Dis Child 2018:archdischild-2017-313653.

13 Ladak LA, Hasan BS, Gullick J, et al. Health-related quality of life in congenital heart disease surgery patients in Pakistan: protocol for a mixed-methods study. BMJ Open 2017:7:e018046.

14 Ferrans CE, Zerwic JJ, Wilbur JE, et al. Conceptual model of health-related quality of life. J Nurs Scholarsh 2005:37:336-42.
15 Warnes CA, Liberthson R, Danielson GK, et al. Task force 1: the changing profile of congenital heart disease in adult life. J Am Coll Cardiol 2001;37:1170-5.

16 Jacobs JP, Franklin RC, Jacobs ML, et al. Classification of the functionally univentricular heart: unity from mapped codes. Cardiol Young 2006:16:9-21.

17 Brander G, Rydell M, Kuja-Halkola R, et al. Perinatal risk factors in Tourette's and chronic tic disorders: a total population sibling comparison study. Mol Psychiatry 2018;23.

18 Manlhiot C, Knezevich S, Radojewski E, et al. Functional health status of adolescents after the Fontan procedure -- comparison with their siblings. Can I Cardiol 2009:25:S294-S300.

19 Soper D. Statistics calculator. 2017. 2017 http://www.danielsoper.com/statcalc3/calc. aspx?id=1 (Accessed 01 April, 2017).

20 Uzark K, Zak V, Shrader P, et al. Assessment of quality of life in young patients with single ventricle after the fontan operation. J Pediatr 2016;170:166-72.

21 Uzark K, Jones K, Slusher J, et al. Quality of life in children with heart disease as perceived by children and parents. Pediatrics 2008;121:e1060-e1067.

22 Idorn L, Jensen AS, Juul K, et al. Quality of life and cognitive function in Fontan patients, a population-based study. Int I Cardiol 2013;168:3230-5.

23 Knowles RL, Day T, Wade A, et al. Patient-reported quality of life outcomes for children with serious congenital heart defects. Arch Dis Child 2014;99:413-9.

24 Varni JW, Limbers CA, Sorensen LG, et al. PedsQLTM Cognitive Functioning Scale in pediatric liver transplant recipients: feasibility, reliability, and validity. Qual Life Res 2011:20:913-21.

25 Trust MR. The Pedsqltm measurement model for the pediatric quality of life inventoryTM. -accessed 9 Jan 2018 http://www.pedsql.org/conditions.html.

26 Varni JW, Limbers C, Burwinkle TM. Literature review: health-related quality of life measurement in pediatric oncology: hearing the voices of the children. J Pediatr Psychol 2007;32:1151-63.

27 Chen S, Ravallion M. Absolute poverty measures for the developing world, 19812004. Proc Natl Acad Sci U S A 2007:104:16757-62.

28 Jenkins KJ. Risk adjustment for congenital heart surgery: the RACHS-1 method. Semin Thorac Cardiovasc Surg Pediatr Card Surg Annu 2004;7:180-4.

29 Nakagawa S, Cuthill IC. Effect size, confidence interval and statistical significance: a practical guide for biologists. Biol Rev Camb Philos Soc 2007;82:591-605.

30 Field AP. Discovering Statistics using SPSS. 4 edn. London: Sage Publications, 2013.

31 Chatfield C, Zidek J, Lindsey J. An introduction to generalized linear models: Chapman and Hall/CRC, 2010

32 Neal AE, Stopp C, Wypij D, et al. Predictors of health-related quality of life in adolescents with tetralogy of Fallot. J Pediatr 2015;166:132-8

33 Schaefer C, von Rhein M, Knirsch W, et al. Neurodevelopmental outcome, psychological adjustment, and quality of life in adolescents with congenital heart disease. Dev Med Child Neurol 2013;55:1143-9.

34 Werner $\mathrm{H}$, Latal B, Valsangiacomo Buechel $\mathrm{E}$, et al. Health-related quality of life after open-heart surgery. J Pediatr 2014;164:254-8.

35 Mocumbi AO, Lameira E, Yaksh A, et al. Challenges on the management of congenital heart disease in developing countries. Int J Cardiol 2011;148:285-8.

36 Fraisse A, Jais X, Schleich JM, et al. Characteristics and prospective 2-year follow-up of children with pulmonary arterial hypertension in France. Arch Cardiovasc Dis 2010:103:66-74

37 Yurgelun-Todd D. Emotional and cognitive changes during adolescence. Curr Opin Neurobiol 2007:17:251-7.

38 Hullmann SE, Wolfe-Christensen C, Meyer WH, et al. The relationship between parental overprotection and health-related quality of life in pediatric cancer: the mediating role of perceived child vulnerability. Qual Life Res 2010;19:1373-80.

39 Wei H, Roscigno Cl, Swanson KM. Healthcare providers' caring: Nothing is too small for parents and children hospitalized for heart surgery. Heart Lung 2017:46:166-71

40 Sen AC, Morrow DF, Balachandran R, et al. Postoperative infection in developing world congenital heart surgery programs. Circulation 2017;10:e002935.

41 Moons P, Kovacs AH, Luyckx K, et al. Patient-reported outcomes in adults with congenital heart disease: Inter-country variation, standard of living and healthcare system factors. Int I Cardiol 2018:251:34-41.

42 Maughan ED, Cowell J, Engelke MK, et al. The vital role of school nurses in ensuring the health of our nation's youth. Nurs Outlook 2018:66. 